\title{
iTRAQ-Based Quantitative Proteome Analysis Insights into Cold Stress of Winter Turnip Rapa (Brassica rapa L.) Grown in the Field
}

\section{Zaoxia Niu}

Gansu Agricultural University

Lijun Liu

Gansu Agricultural University

\section{Yuanyuan Pu}

Gansu Agricultural University

Li Ma

Gansu Agricultural University

Junyan Wu

Gansu Agricultural University

\section{Fangdi Hu}

Gansu Agricultural University

Yan Fang

Gansu Agricultural University

Xuecai Li

Gansu Agricultural University

Wancang Sun ( $\sim$ 18293121851@163.com )

Gansu Agricultural University

Chunsheng Bai

Gansu Agricultural University

Wangtian Wang

Gansu Agricultural University

\section{Research Article}

Keywords: Cold Stress, Winter Turnip Rapa, Brassica rapa L.

Posted Date: June 18th, 2021

DOI: https://doi.org/10.21203/rs.3.rs-590152/v1 
License: (c) (i) This work is licensed under a Creative Commons Attribution 4.0 International License. Read Full License 


\title{
iTRAQ-Based Quantitative Proteome analysis insights
}

\section{into cold stress of Winter Turnip Rapa (Brassica rapa L.)}

\author{
grown in the field \\ Zaoxia Niu ${ }^{1,2}$, Lijun Liu ${ }^{2}$, Yuanyuan $\mathrm{Pu}^{1,2}$, Li Ma ${ }^{2}$, Junyan $\mathrm{Wu}^{1,2}$, Fangdi $\mathrm{Hu}^{1,2}$, Yan Fang ${ }^{2}$, Xuecai \\ $\mathrm{Li}^{1,2}$, and Wancang Sun ${ }^{1,2}, *$, WangtianWang ${ }^{1}$, Chunsheng Bai ${ }^{1}$ \\ ${ }^{1}$ College of Agronomy, Gansu Agricultural University, Lanzhou 730070, China \\ ${ }^{2}$ Gansu Provincial Key Laboratory of Aridland Crop Science, Gansu Agricultural \\ University, Lanzhou 73000, China
}

\begin{abstract}
Winter Turnip rapa (Brassica rapa L.) is a major oilseed crop in Northern China, where its production was severely affected by chilling and freezing stress. Previous studies have demonstrated that differentially accumulated proteins (DAPs) were expressed in roots and leaves under control chilling stress. In this study, the isobaric tag for relative and absolute quantification (iTRAQ) technology was performed to identify DAPs under freezing stress. Two winter rapaseed varieties, Longyou 7 (cold-tolerant) and Lenox (cold-sensitive), were used to investigated morphological, physiological, cell and protein levels in the shoot apical meristem (SAM) of field-grown Brassica rapa to reveal the molecular mechanisms of cold stress tolerance. Compared to Lenox, Longyou 7 had a lower SAM of height, higher collar diameter. The level of malondialdehyde (MDA), SAM of height and IAA content were repressed. At the same time, compared to Lenox, the soluble sugars (SS) content, superoxide dismutase (SOD) activity, peroxidase(POD)activity, soluble protein (SP) content and collar diameter increased in Longyou 7. In total, we identified 6330 proteins, among this, 98 DAPs were expressed in $\mathrm{L} 7 \mathrm{CK} / \mathrm{Le} \mathrm{CK}, 107 \mathrm{DAPs}$ were expressed in $\mathrm{L} 7 \mathrm{~d} / \mathrm{Le} \mathrm{d} 183 \mathrm{DAPs}$ were expressed in Le d /Le CK, 111 DAPs were expressed in L7 d /L7 CK. Quantitative real-time PCR (RT-qPCR) analysis of the coding genes for seventeen randomly selected DAPs were performed for validation. These DAPs were identified from the two winter rapa seed cultivars involved in the biological process, cellular component and molecular function analysis, which revealed glutathione transferase activity, carbohydrate-binding, and glutathione binding, glutathione metabolic process and response IAA were closely associated with the cold stress response. Some cold-induced proteins, such as glutathione S-transferase phi 2(GSTF2), might play essential roles cold acclimation in Brassica rapa of SAM. Our work will help to provide valuable information for responding to the cold stress in Brassica rapa $\mathbf{L}$.
\end{abstract}

\section{Introduction}

Cold stress, including chilling $\left(0-15^{\circ} \mathrm{C}\right)$ and freezing $\left(<0{ }^{\circ} \mathrm{C}\right)$ temperatures, dramatically affects plant growth, yield, and quality of crop species. It is one of the major threats to crop production in northwestern China[1, 2]. Plants adopted several strategies to develop complicated and effective coldresponsive mechanisms to survive cold stress, such as the level of chaperones and antioxidants variations in their leaf tissue structure, maintaining osmotic balance by altering membrane structure, and the activation of cold-related genes[3,4]. Some plants abandon their cold sensitive structure to protect themselves in winter, such as above-ground or avoid freezing injury by shrinking vegetative organs into underground organs. Although the way in which plants perceive and respond to temperature cues is not well understood, wheat winter survival involves two important evolutionary adaptive mechanisms: cold acclimation and vegetative/ reproductive transition[5, 6]. The most important growth site in the nutritional compared with reproductive transformation is the SAM, it play an important role in plant growth. Meristem function is essential for local auxin synthesis, and auxin biosynthesis genes also show specific as well[7]. So, cold stress of molecular mechanisms will shed light on the regulatory mechanisms for cold acclimation in Brassica rapa and provide an effective approach to select targeted candidate genes for manipulation and and/or cross-breeding of agronomic[8]. Our study will shed light on regulatory mechanisms of cold acclimation and provide an effective approach to select targeted 
candidate genes for manipulation and cross-breeding of agronomic in $B$ rapa.

iTRAQ is a gel free mass spectrometry technique for the corresponding protein. It applies isobaric amine specific tags to compare peptide intensities between samples, followed by inferring quantitative values for the corresponding proteins[9]. iTRAQ labelling combined with two-dimensional liquid chromatography-tandem mass spectrometry(2D-LC-MS compared with MS) is a high through put quantitative proteomics technique widely used in identifying and quantifying cellular metabolic changes in proteome-related analysis[10]. This technique has been successfully applied to identify plant protein responses to stress in various plants such as Vitis amurensis[11], Triticum aestivum[12], Gossypium hirsutum L[13], Lycopersicon esculentum Mil[14], Brassica rapa L[15], Spica Prunellae[16]. Differential proteomics analysis focused on screening and identifying differences and changes in different species or growth stages, revealing and validating proteomics changes.

Brassica winter rapa (Brassica rapa L.) is an oil crop that can survive the winter in the cold and arid region of northern China and is one of the effective surface cover crops in winter and spring. It has significant ecological and economic benefits in agricultural production[17]. However, the extremely low temperature in northern China negatively influences the regional distribution and safe production of winter rapa in winter. Previous studies have shown that strong cold-resistant plants show that their leaves crawl and grow close to the ground in morphology. Before winter, organic matter is preferentially distributed to the root, storing enough organic matter and establishing an extensive root system, guaranteeing its safe overwintering and vegetativeness construction after winter. Previously, our research team has used high-throughput sequencing technology and successfully identified cold-stressresponsive microRNAs(miRNAs)[18], differentially expressed genes(DEGs)[19] and differentially accumulated proteins(DAPs)[20] in roots and leaves of $B$. rapa. The SAM depression and bulge in the seedling stage might be associated with their strong cold resistance. The functions of SAM target protein regulations have not yet been understood. The objectives were to explore the relationship between SAM-target proteins regulations and their relationship to cold stress tolerance. The proteins identified can be used to inform breeding programs and improve our understanding of the molecular mechanisms underlying cold resistance.

\section{Results}

Physiological development and cold tolerance. Longyou 7 and Lenox was used to study the morphologyical and physiological differences at the seedling stage under cold stress. Lenox had an upright petiole with several leaves growing in the leaf positions. However, Longyou 7 was always opposite (Fig 1A). When growing in an open field, the height of SAM levels was measured at the different growth stage. Lenox of average height of SAM since the first sampling date and displayed a high apical meristem at the next stage. Compared with the first sampling date (CK), Lenox of average height of SAM was significantly increased in the the next stage, Longyou 7 of average height of SAM was increased in the next stage. Compared with Longyou 7 of average height of SAM, Lenox of average height of SAM was significantly higher. Compared with the first sampling date (CK), Longyou 7 of collar diameter was significantly increased in the the next stage (d), Longyou 7 of collar diameter was increased in the next stage. Compared with Longyou 7 of collar diameter, Lenox of collar diameter was significantly higher (Fig 1B). Compared with the L7 $d$ and Le d, the level of SOD, POD activity and soluble protein content significantly decreased in the L7 CK and Le CK samples. The MDA content significantly increased in $\mathrm{L} 7 \mathrm{CK}$ and Le CK. Under freezing treatment $\left(0^{\circ} \mathrm{C}\right.$ and $\left.-11^{\circ} \mathrm{C}\right)$, the MDA level of Le CK and Le d was significantly higher than that of L7 CK and L7 d. Still, other physiological indexes of Le CK and Le $d$ were lower than those of L7 CK and L7 d (Fig 1C ). These results suggested that compared with Lenox, Longyou 7 had lower SAM but higher tolerance to freezing stress. Strong coldresistant varieties such as Longyou 7 were found have a hollow SAM in the samples collected from field experiments, which were $5-10 \mathrm{~cm}$ below the soil surface. Thus the SAM always located in the moist soil under a relatively stable temperature, which is beneficial for winter $B$. rapa to the overwinter. These findings showed the SAM seedling stage have been related to cold tolerance. 

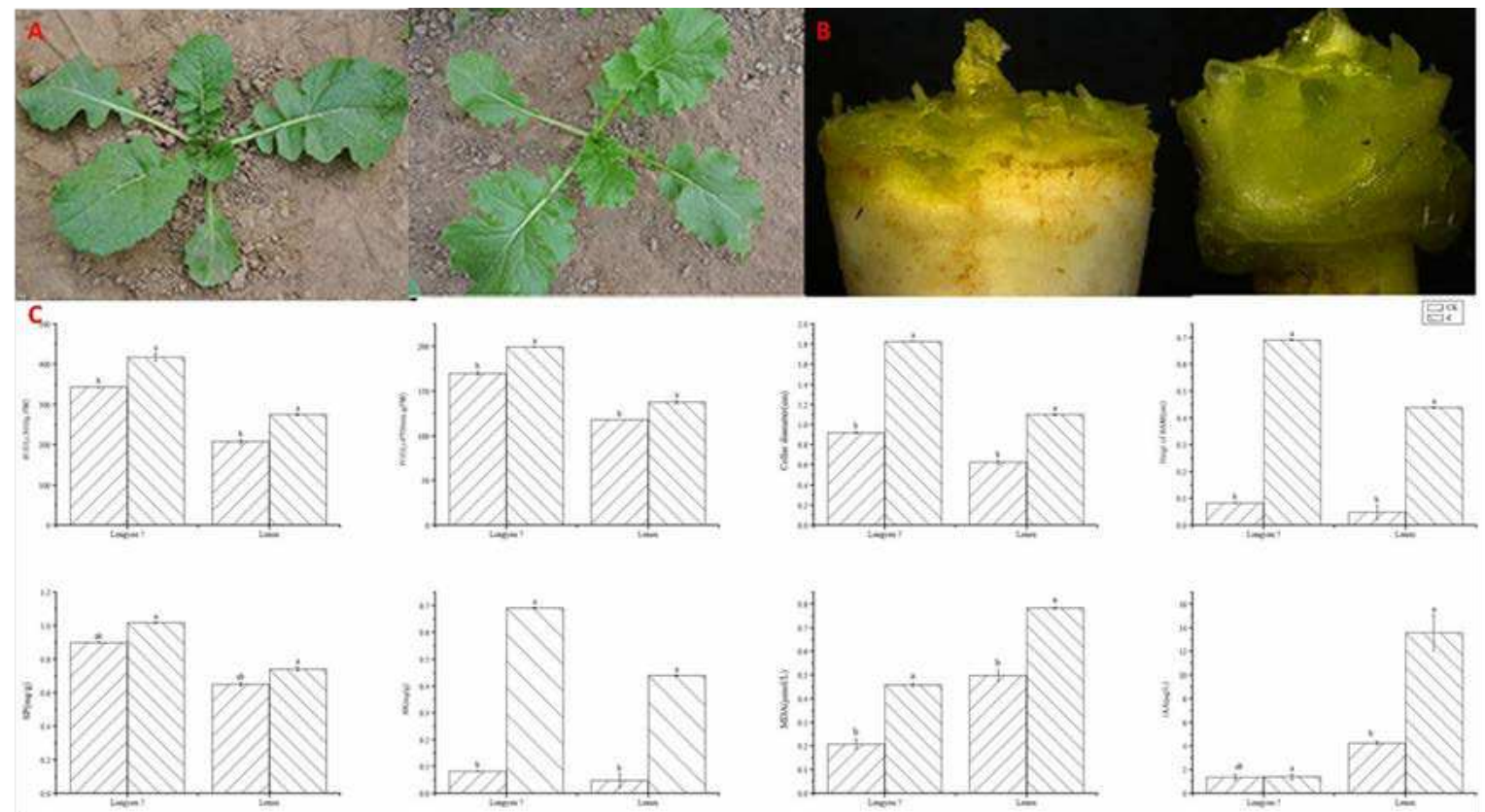

Figure 1. (A) The growth characteristics of Longyou 7 and Lenox. (B) The SAM and collar of the morphology of Longyou 7 and Lenox. (C) Morphological and physiological indicators of Longyou 7 and Lenox under overwinter.The majuscules indicated a significant difference $(p<0.01)$ for the freezing stress-treated samples compared with chilling stressed samples. Values are means and SD of three biological replicates, each calculated from the mean of three technical replicates.

iTRAQ-Based quantitative proteome analysis. Previous RNA-seq and iTRAQ-based quantitative proteome analysis applied on leaves and roots tissues were limited to obtaining relative differences of the genes or proteins expression under controlled environment. Our study had demonstrated that DAPs used to evaluate relative differences of protein expression as cold acclimation progressed under field conditions from early autumn to winter. The field trials were designed to capture the big picture of global changes of the proteomics during cold acclimation in Longyou 7 and Lenox. iTRAQ-based quantitative proteome analysis was performed in Longyou 7 and Lenox. The analysis of protein was performed using 8-plex iTRAQ-based comparative proteome analysis. Three independent biological replicates were simultaneously performed. After using Mascot to search against the Brassica-rapa1.0 database. In total, 6328 protein were identified (Supplementary Table S1).

iTRAQ quantitative identified DAPs. Based on fold change $(F C)>1.5(p<0.05)$, DAPs were up accumulated, $\mathrm{FC}<0.8$ ( $p<0.05$ ), DAPs were down accumulated. Here, up or down accumulated proteins were determined using the Longyou 7 and Lenox. The Venn diagram reflected the DAPs in two winter rapa seed varieties. 76 DAPs were found in between the Le $\mathrm{d} / \mathrm{Le} \mathrm{CK}$ and L7 $\mathrm{d} / \mathrm{L} 7 \mathrm{CK}$ and 48 DAPs were found in both the L7 d /Le $d$ and L7 CK /Le CK (Figure 2A and 2B). Of these, there were 35 DAPs unique to $L 7 \mathrm{~d} / \mathrm{L} 7 \mathrm{CK}$. This was consistent with the results of the principal component analysis (PCA) of the three biological replicates, which indicated that the three biological replicates of each sample had a good repeatability, L7 d /L7 CK as well as Le d /Le CK had a great difference (Figure 2C ). As shown in Figure2D, 111 DAPs were expressed in L7 d /L7 CK with 57 up accumulated and 54 down accumulated DAPs (Supplementary Table S2). 98 DAPs were expressed in L7 CK /Le CK with 29 up accumulated and 69 down accumulated DAPs (Supplementary Table S3). 183 DAPs were expressed in Le d /Le CK with 80 up accumulated and 103 down accumulated DAPs (Supplementary Table S4). 107 DAPs were expressed in L7 d /Le d with 28 up-accumulated and 79 down accumulated DAPs (Supplementary Table S5). 
A

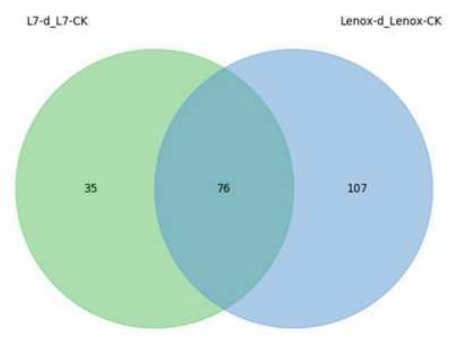

C

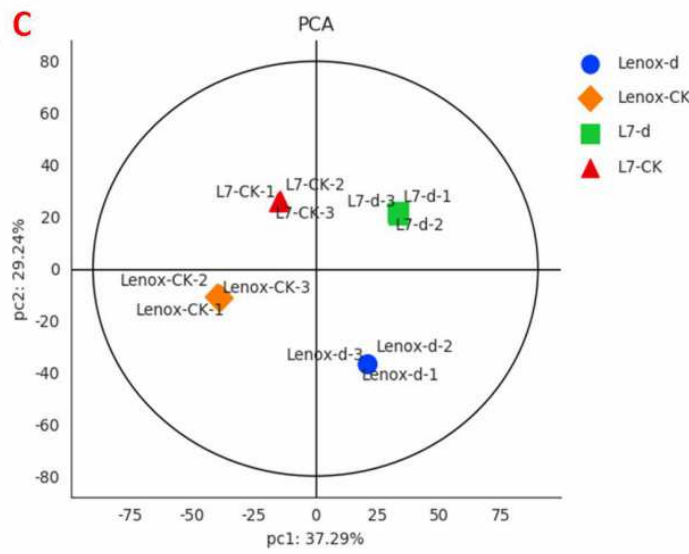

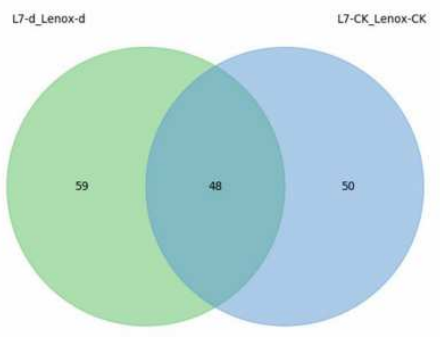

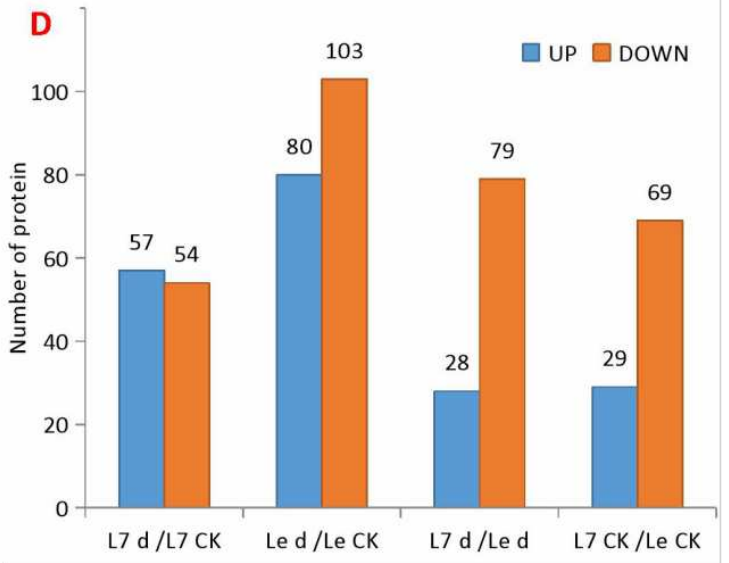

Figure 2. (A) Venn diagrams of DAPs identified by iTRAQ between L7 d / L7 CK and Le d / Le CK. (B) Venn diagrams of DAPs identified by iTRAQ between different $\mathrm{L} 7 \mathrm{~d} / \mathrm{Le} \mathrm{d}$ and L7 CK/ Le CK. L7 d / L7 $\mathrm{CK}$ is the protein abundance ratio of $\mathrm{L7} \mathrm{d}$ compared with $\mathrm{L} 7 \mathrm{CK}$, Le $\mathrm{d} / \mathrm{Le} \mathrm{CK}$ is the protein abundance ratio of $\mathrm{Le} d$ compared with $\mathrm{Le} C K, \mathrm{~L} 7 \mathrm{CK} / \mathrm{Le} \mathrm{CK}$ is the protein abundance ratio of $\mathrm{L} 7 \mathrm{CK}$ compared with $L e C K$, and $L 7 d / L 7 C K$ is the protein abundance ratio of $L 7 d$ compared with $L 7$ CK. (C) PCA plots of the proteome in three biological replicates from cold-stressed and freezing-stressed winter turnip rapa. (D) Number of up- and down-accumulated proteins among different comparison groups.

Classification of cold reposive DAPs. To obtain the function of the DAPs all quantified proteins were searched through the UniProt-GOA database(https://www.ebi.ac.uk/GOA/), and gene ontology (GO) annotation(http:// www. panth erdb. org/). In this study, GO annotated 2669 identified proteins. Cellular component, biological process and molecular function were assigned among DAPs based on the results of GO analysis (Supplementary Table S6). GO analysis was shown in L7 d /L7 CK(Fig 3). Compared with Le d /Le CK, L7 d /L7 CK was unique to response to phenylpropanoid (GO: 0080184), and starch catabolic process GO: 0005983) in up the top 10 biological processes. L7 d /L7 CK was unique to response to sucrose (GO: 0009744), negative regulation of cysteine-type endopeptidase activity (GO: 2000117), phosphoenolpyruvate transport (GO: 0015714), regulation of photosynthesis (GO: 0010109) and triose phosphate transmembrane transport (GO: 0035436) in the down top 10 biological processes. Of these, starch catabolic process was both down regulated and in L7 d /L7 CK. 

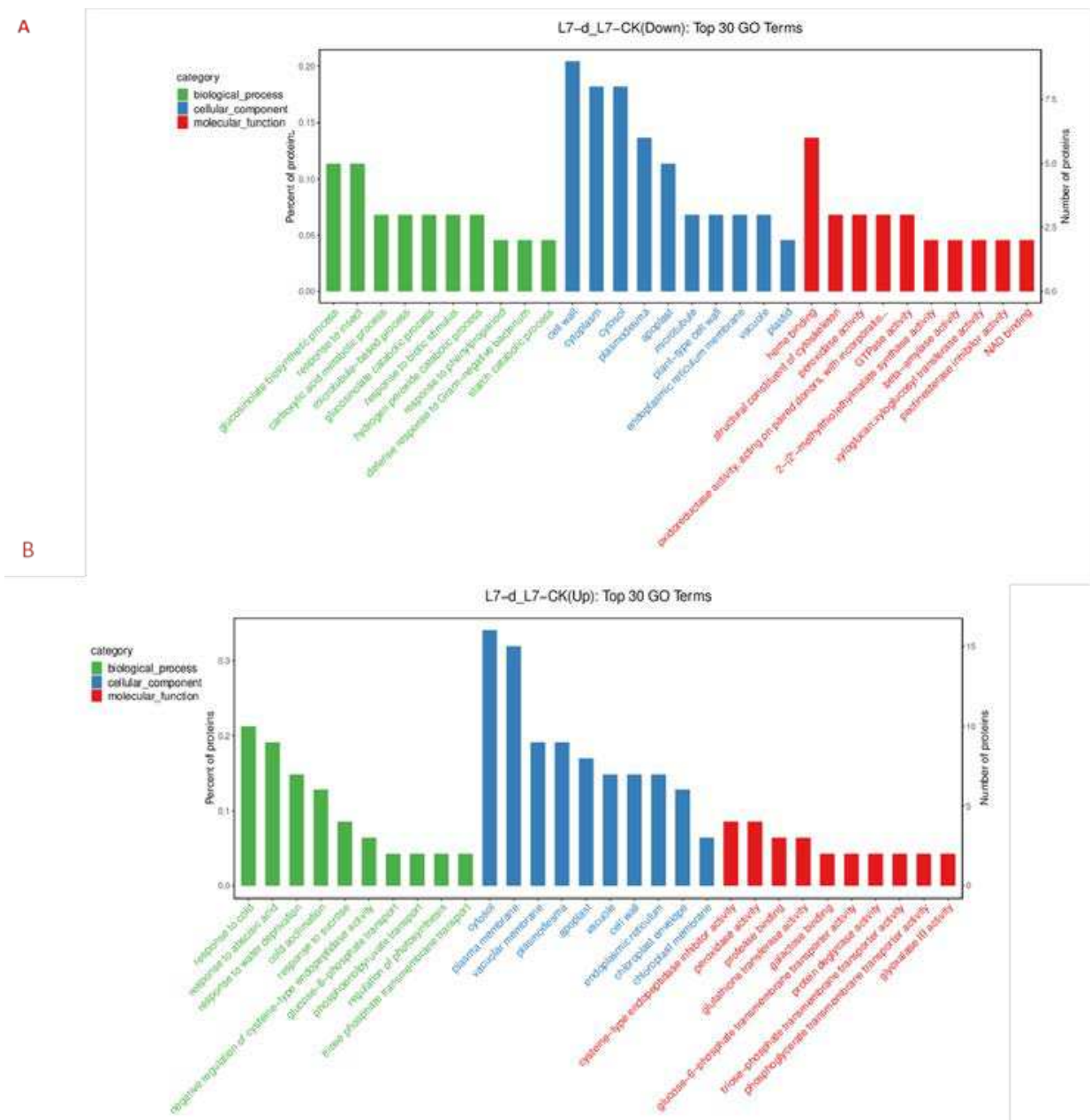

Figure 3. (A) Gene ontology (GO) enrichment analysis of down regulated DAPs in L7 d /L7 CK. (B) GO enrichment analysis of up regulated DAPs in L7 d/L7 CK.

To further characterize the functions of the DAPs, KEGG pathway mapping was also performed. Only significantly enriched categories $(p<0.05)$ were selected (Supplementary Table S7). KEGG enrichment analysis displayed the pathways of DAPs between L7 d /Le d. DAPs in up regulated from $L 7 \mathrm{~d} / \mathrm{Le} \mathrm{d}$ was found to been enriched in glutathione metabolism (brp00480), thiamine metabolism (brp00730), starch and sucrose metabolism (brp00500), tyrosine metabolism(brp00350), fatty acid degradation(brp00071), glycolysis / gluconeogenesis (brp00010), oxidative phosphorylation (brp00190), phenylpropanoid biosynthesis (brp00940) and protein processing in endoplasmic reticulum (brp04141). 41 DAPs down regulated from $L 7 \mathrm{~d} / \mathrm{Le} \mathrm{d}$ was found to been enriched in glutathione metabolism(brp00480), linoleic acid metabolism(brp00591), alpha-Linolenic acid metabolismbrp(00592), pentose phosphate pathway(brp00030), phenylpropanoid biosynthesis(brp00940), carotenoid biosynthesis(brp00906), alanine, aspartate and glutamate metabolism(brp00250), glycosphingolipid biosynthesis-globo and isoglobo series (brp00603).

L7 d /L7 CK compared with Le d /L e CK, L7 d /L7 CK was unique to the histidine metabolism (brp00340), pentose and glucuronate intercnversions(brp00040), pyrimidine metabolism(brp00240), glycine, serine and threonine metabolism(brp00260), pyruvate metabolism(brp00620) and RNA degradation(brp03018) in up enrichment top 20. L7 d /L7 CK was unique to the arachidonic acid metabolism (brp00010) in down enrichment top 20 (Fig 4). 


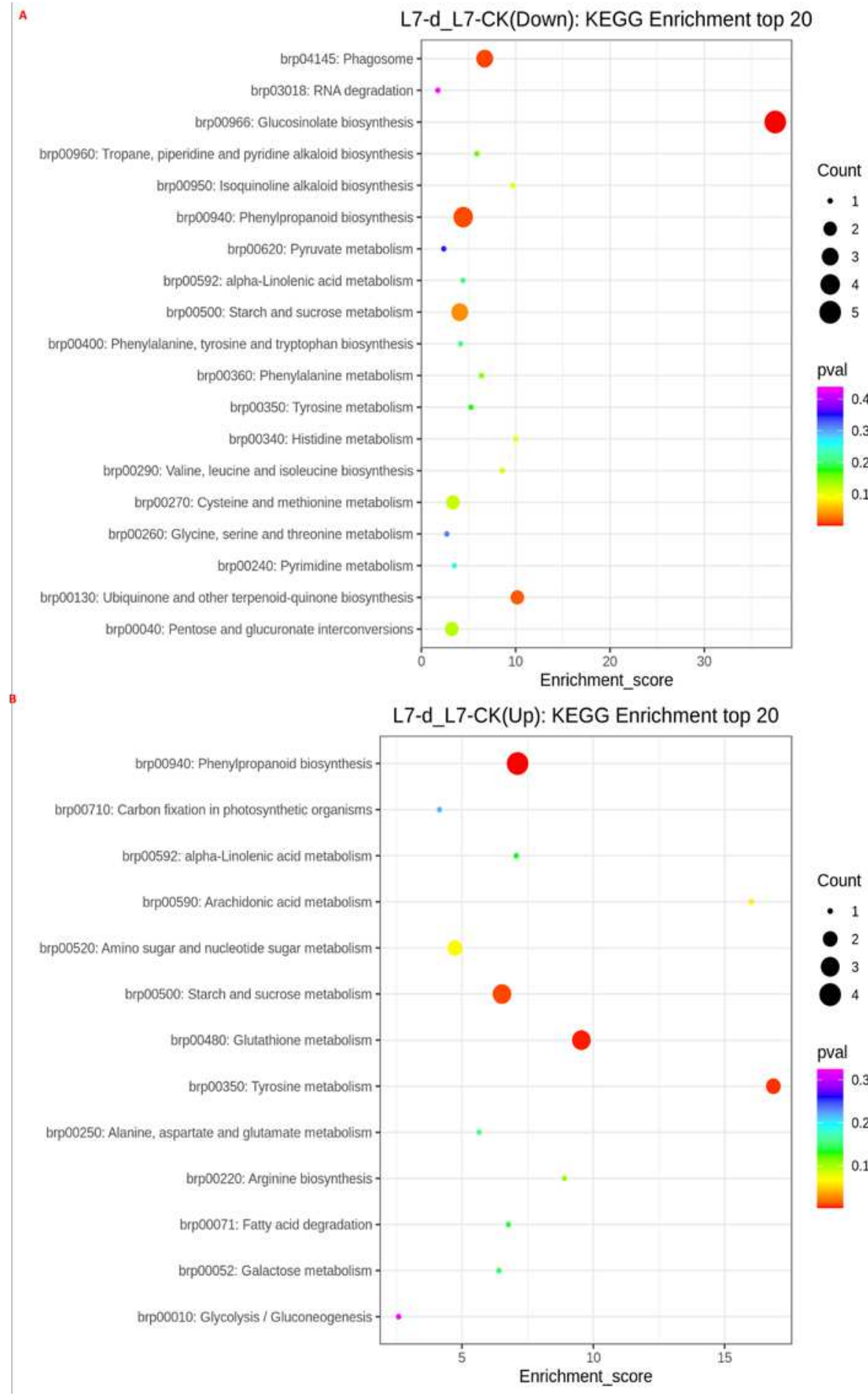

Figure 4 . (A) Kyoto Encyclopedia of Genes and Genomes (KEGG) pathway enrichment analysis of down regulated DAPs in L7 d / L7 CK. (B) KEGG pathway enrichment analysis of up regulated in L7 d/L7 CK.

qRT-PCR revealed expression analysis of selected DAPs. Transcript expression levels were detected by qRT-PCR (Fig 5). Seventeen genes were randomly selected to validate the reliability of the translational level data. Among those, seventeen genes displayed almost similar expression patterns to their protein levels in two cultivars, such as BCPI-2(cysteine protease inhibitor, putative/cystatin, putative), LOX2 (lipoxygenase 2), GSTF2 (glutathione S-transferase 2), CYP83A1 (cytochrome P450 83A1), AMY1 (alpha-amylase-like), MAM1 (methylthioalkylmalate synthase 1), ATTI1(serine-type endopeptidase inhibitor), RAB18(responsive to ABA 18), PRXR1(peroxidase 1), DHAR1 (dehydroascorbate reductase), EXL2 (exordium like 2), ATGSTF3(glutathione S-transferase F3), 
ATGSTF11 (glutathione S-transferase F11), BAM5 (beta-amylase 5). However, MLP 328(MLP-like protein 328) and ERD10 (early reponsive to dehydration 10) was opposite with the protein abundances probably resulted from various posttranslational modifications.

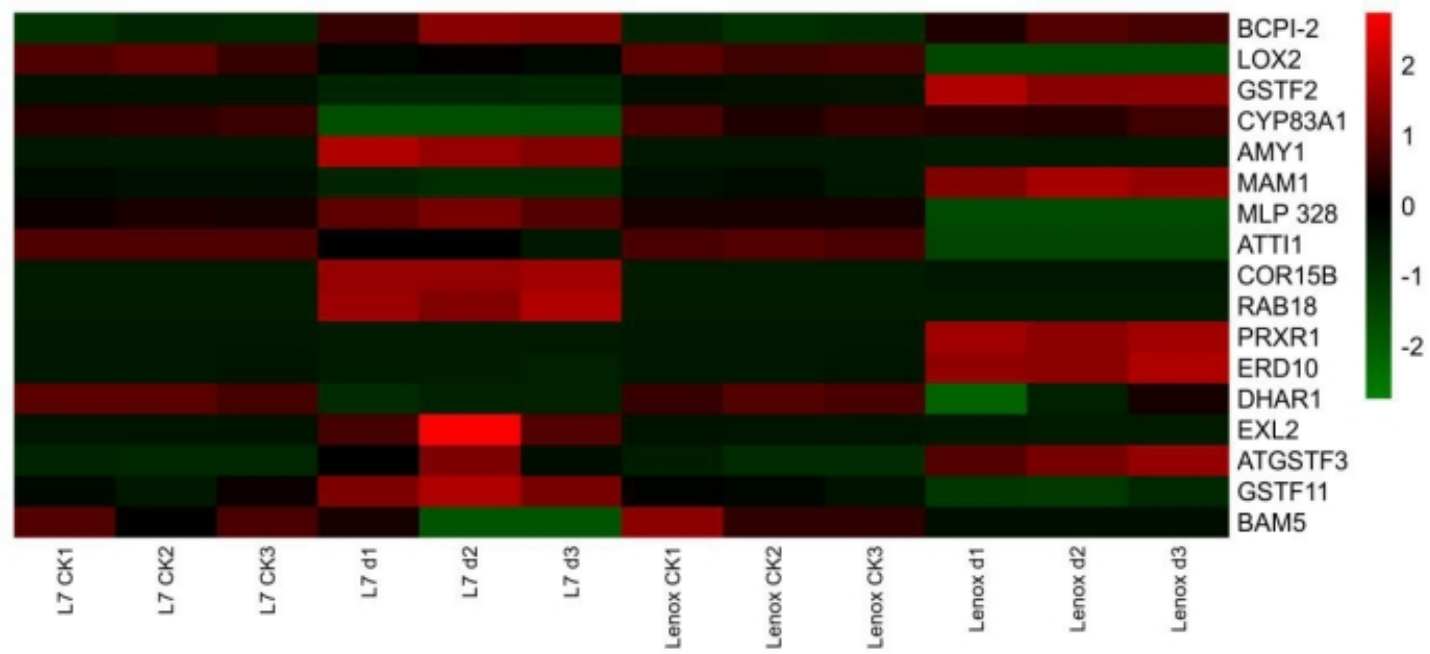

Fig 5. RT-qPCR analysis of genes related to DAPs. Transcript abundance was calculated according to the difference in cycle threshold values between the target gene and $\beta$-actin transcripts normalized by the $2-\triangle \triangle C T$ method.

\section{Discussion}

Proteomic analysis has identified many differentially accumulated proteins mainly related to plant secondary metabolism in germinated seeds under cold stress. In this study, a large number of cold stress-responsive proteins were identified in winter turnip rapa. Previously several candidate genes has been identified by EST analysis which may be involved in the cold stress response in winter turnip rapa. However, the mechanisms underlying the effect of cold stress on SAM are largely unknown. Therefore, that physiological and proteomic analyses were performed in this study to examine the mechanism of the increased freezing tolerance in winter tunip rapa.

Freezing Stress Affects Morphology, Physiological and Biochemical Changes in Brassica rapa. Freezing stress $\left(<0^{\circ} \mathrm{C}\right)$ harmfully affects plant growth and development, limits their geographic distribution, and significantly reduces agronomic productivity[20]. In the natural cooling process, plants change in morphology, physiology and biochemistry due to low temperature stress, especially freezing stress[21]. The main target of freezing injury is cell membranes. Lipid, peroxide and MDA could reflect the damage of plants under low temperature[22]. The regulation of osmotic adjustment substances such as soluble protein, and free proline could keep the stability of cellular structure and osmotic balance[23]. Meanwhile, proline acts as a cell structure maintainer, a signaling molecule and a ROS scavenger in the response to cold stress, among these, SOD and POD are key factors of antioxidant enzymes under stress[24, 25]. There was an increase in the content of MDA and SP in the CK compared to freezing stress. However, the content of Pro, SOD and POD activity decreased in in the CK compared to freezing stress (Figure 1C). The increase of osmotic adjustment substance content could reduce the freezing point to increase plant cell membrane stabilization and protect membrane integrity during dehydration caused by freezing treatment in B. rapa. The aboveground tissues of winter rapa seed were damaged by low temperatures. Longyou 7 has strong protective enzyme activity and high content of soluble regulators that can remove ROS. These data showed that osmometric and ROS were adjusted in $B$. rapa to adapt to the cold stress.

DAPs involved in lipid metabolism. Several studies have shown that high concentration of unsaturated fatty acids are commonly presented in plants grown under cold stress, thus cold-tolerant plants often have higher unsaturated membrane lipids[26, 27]. Linoleic an alpha-Linolenic acids play key roles in maintaining membrane integrity and fluidity, and have been demonstrated to contribute to the osmotic tolerance in rice[28, 29]. Unsaturated sphingolipids content is reported to be higher in coldtolerant species $[28,30]$. Glycerolipid metabolism is one of the metabolic pathways related to fatty acid degradation. Glycerol plays vital roles in cell energy generation and lipid synthesis[31]. In previous study, lipoxygenase LOX could damage to cell membranes and other cell components[32]. LOX2 was downregulated under cold stress, Linolenate hydroperoxide lyase (CYP74B2) and LOX2 play a role in JA biosynthesis[33, 34]. Cold hardiness increased in S. cerevisiae when acohol dehydrogenase class- 
3(ADH3) was overexpressed[35]. In this study, DAPs were found involve in Linoleic acid metabolism, alpha-Linolenic acid metabolism, glycolipid metabolism, sphingolipid metabolism, and fatty acid degradation. Of these, only fatty acid degradation was up regulated and rest were down regulated in L7 d /Le d. ADH3(Bra033706) was up regulated, LOX2(Bra004057, Bra003526, Bra003526) and CYP74B2(Bra012788) were down regulated in L7 d /Le d. It is known that ADH3 might play an important role in the cold stress of $B$. rapa.

DAPs involved in carbohydrate and energy metabolism. Carbohydrate and energy metabolism are the basis of plant growth, development and morphogenesis [36]. Carbohydrate metabolism occupies the core of energy metabolism since it provides the essential saccharides and energy that plants need[37]. It was reported that beta-amylase 5 (BAM5) might also play a important role in the petunia response to cold stress[38]. Down regulation of alpha-galactosidase $(\alpha-G a l)$ has been shown to increase plant freezing tolerance[39]. The pentose phosphate pathway(PPP) supplies NADPH, an electron donor[40]. Probable 6-phosphogluconolactonase 4(PGL4), an enzyme participating in PPP agreed with the increased needs for ROS scavenging[39]. 1,4-alpha-glucan-branching enzyme2-41(SBE2.1) increased in salt and drought stressed mulberry roots and leaves, are involved in starch biosynthetic metabolism[40]. In this study, $\alpha-G a l ~ 1$ (Bra028660) and PGL 4(Bra009759) were down regulated in L7 d /Le d BAM5(Bra038088) and SBE2.1 (Bra005269) were up regulated in L7 d /Le d. BAM5 and SBE2.1 were involved in the starch and sucrose metabolism. Previous study suggested that many starch grains accumulated in the leaf cells under stress. So, this might provide a new direction for future research in the $B$. rapa under cold stress.

Energy metabolism may play important roles in the development of chilling resistance in plant[41]. Nitrilase 2 (NIT 2) promotes nitrilase biosynthesis, and nitrilase leads to increased IAA content in Arabidopsis under cold stress[42]. Cytochrome oxidase subunit 6b-1(COX 6 B) was downregulated in rice under salt stress. Chlorophyll a-b binding protein increased in mulberry leaves under salt-drought stress conditions[43]. In our study, DAPs participated in energy metabolism, including nitrogen metabolism, photosynthesis-antenna proteins, oxidative phosphorylation were identified in L7 d/Le d. Our results showed that the expression levels of chlorophyll a-b binding protein CP 24 (Bra026745) and NIT 2 (Bra035006) increased, while and COX6B (Bra031391) decreased in L7 d / Le d. Our results also indicated that IAA content of the Longyou 7 higher compared to Lenox in the SAM of the B. rapa. Therefore the focus of our future research will be on NIT 2 and auxin in the $B$. rapa under cold stress.

DAPs involved in amino acid metabolism. It is reported that freezing stress response amino acids metabolic pathway in Eriobotrya japonica and Populus tomentosa under cold or freezing conditions[44, 45]. Our study demonstrated that several amino acid metabolic pathways were identified after freezing stress. Seventeen DAPs were annotated inclduing Glutathione metabolism, Cysteine and methionine metabolism, Alanine, aspartate and glutamate metabolism, Arginine biosynthesis, Tryptophan metabolism, Glycine, serine and threonine metabolism and Tyrosine metabolism. Glutathione Stransferases (GSTs) are a major family of detoxification enzymes that are important in protecting plants against oxidative damages. Auxin induced the expression of several glutathione S-transferases (GSTs) such as AtGSTF 2, so GSTF 2 is up regulated might be linked to stress-mediated growth responses[46]. Our results showed that bigger of SAM indicated relatively high levels of IAA content, while a relatively smaller SAM indicates relatively low levels of IAA content. so auxin content and thus was related auxin content in relatively high of SAM. Our results showed that five GSTs (Bra000875, Bra021673, Bra000876, Bra036259, Bra012409) and glutathione S-transferase DHAR 1 (Bra025749) were down regulated, while GSTU (Bra012422, Bra000474) and GSTs (Bra032010) were up regulated in L7 d /Le d. In addition, antioxidant enzymes played important roles in the removal of excess ROS to reduce oxidative stress by GSTs. the activities of a number of antioxidant enzymes, such as SOD and POD can remove ROS. The increased accumulation of these proteins indicated that plant cells initiated their antioxidant mechanisms to maintain redox homeostasis and resist cold stresses.

DAPs involved in translation and biosynthesis of secondary metabolite. Posttranslational modification (PTM) in Brassica juncea cold stress systems include S-nitrosylation[46]. Post-translational modification that confers the glutathione S-transferase (GST) under oxidative stress [47]. The biosynthesis of secondary metabolites are associated with their involvement in tolerance by cold stress in plant[48]. Secondary metabolites are involved in plant responses to abiotic stresses and provide a valuable contribution to the antioxidant activity of plant tissues [49]. Protein disulfide isomerase (PDI) gene was over-expressed that can enhance heat stress [50]. Tropinone reductase (TR1) significantly increased the low temperature in Arabidopsis [51]. ADH3(Bra033706) was up regulated and PDI(Bra020239) was down regulated in Longyou-7 d / Lenox d. Tropin one reductase homolog (Bra039974) was involved in 
biosynthesis of other secondary metabolites and folding, sorting and degradation. GST was involved in posttranslational modification in Longyou-7 d /Lenox d. Our study showed its great potentials in improving the genetics of low temperature tolerance in plants.

\section{Conclusions}

In this study, two winter cultivars (Longyou 7 and Lenox) with different phenotypes for cold stress were used to analyze molecular mechanisms for cold response. Base on proteomics data, major proteins involved in metabolic pathways through accumulating differentially accumulated proteins in both winter turnip rapa varieties under cold stress. DAPs were identified at treatment between Longyou 7 and Lenox. In addition, based on the functional analysis, we concluded that DAPs involved in amino acid metabolism, carbohydrate and energy metabolism, lipid metabolism, translation and biosynthesis of secondary metabolites, which showed that freezing stress greatly changed the survival of winter turnip rapa. In summary, these findings serves as our understanding of the molecular mechanisms in freezing tolerance and increases protein resource for rapa seed for freezing tolerance breeding in winter turnip rapa.

\section{Methods}

Plant materials, field, sample collection. Two different winter turnip rapa cultivars were used in this study Longyou 7 and Lenox from Gansu Agricultural University (Gansu, China). Both were grown in normal agronomic field trials at Gansu Research Center of Rapaseed Engineering and Technology in Lanzhou, China. All method was performed in accordance with the relevant guidelines and regulations. Three replicates were prepared for each cultivar in the field. The field-collected tissue were used periodically for cold hardiness and morphological measurements form October 13 to December 16, when daily average temperature had reached $0^{\circ} \mathrm{C}$. The daily average temperature is the mean value of the lowest temperature in 15 days. Each sampling point, plants were collected in the field between 10:30 a.m. and 11:30 a.m. to minimize circadian rhythm effect. Data was collected daily at average daily minimum temperature (Tmin) from October to December in 2018. The Tmin was $0^{\circ} \mathrm{C}$ On October 13 , December 16 was $-11^{\circ} \mathrm{C}$. The sample for analysis were named, Le CK (sample of "Lenox" at $0^{\circ} \mathrm{C}$ October 13), Le d (SAM of "Lenox" at $-11^{\circ} \mathrm{C}$ ), L7 CK (SAM of "Longyou 7" at $0{ }^{\circ} \mathrm{C}$ ), and L7 d (SAM of "Longyou 7" at $11^{\circ} \mathrm{C}$ ). To provide sufficient tissue for RNA extraction, SAM (less than a $1 \mathrm{~cm}$ long section at the base of the crown) was collected from four to six plants in each point and replicate. Samples were flash frozen in liquid nitrogen and stored in a $-80^{\circ} \mathrm{C}$ freezer.

Morphological and physiological analyses. The samples were performed three times with similar results. POD activity[51], CAT activity[52], SS content[53], IAA content[54], SP and MDA content[55] were analyzed as indicators of physiological response. The majuscules indicated a significant difference $(p<$ 0.01 ) for the freezing stress-treated samples compared with chilling stressed samples. Values are means and SD of three biological replicates, each calculated from the mean of three technical replicates. Protein extraction. iTRAQ analysis were sent to Shanghai Luming biology (Shanghai, China). Three biological replicates were used for iTRAQ-based comparative proteomics analysis. Approximately 500 $\mathrm{mg}$ from each biological replicate was ground into powder using liquid nitrogen and dissolved (vortex blending) with $500 \mathrm{Ml}$ extraction buffer. The samples were ground at $60 \mathrm{~Hz}$ for $2 \mathrm{~min}$. Then supplemented with extraction buffer for $1 \mathrm{~mL}$ and mixed and added with Tris-phenol buffer and mixed for $30 \mathrm{~min}$ at $4{ }^{\circ} \mathrm{C}$. The homogenates were centrifuged at $7100 \times \mathrm{g}$ for $10 \mathrm{~min}$ at $4{ }^{\circ} \mathrm{C}$. The supernatant was collected, added for five volumes of $0.1 \mathrm{M}$ cold ammonium acetate methanol buffer and stored at $-20^{\circ} \mathrm{C}$ overnight. The homogenates were centrifuged at $12,000 \times \mathrm{g}$ for $10 \mathrm{~min}$ to collect precipitations. The methanol was replaced with acetone to remove the methanol, and the steps used to obtain precipitate were repeated. The samples were centrifuged at $12000 \times \mathrm{g}$ for $10 \mathrm{~min}$ at $4^{\circ} \mathrm{C}$ to collect supernatants. The supernatants were dried at room temperature for $5 \mathrm{~min}$ and dissolved in sample lysate form $2 \mathrm{~h}$. The supernatants were centrifuged again to remove precipitations completely. The protein concentration was quantified using BCA method and the protein purity was detected by SDS-PAGE.15 $\mu$ g proteins of each sample were separated on $12 \%$ SDS-PAGE gel.

Protein digestion, iTRAQ labeling and RP chromatography separation. Protein digestion was performed according to the FASP procedure[56]. IAA was added to the final concentration of $50 \mathrm{mM}$ in the dark for $40 \mathrm{~min}$. The protein solutions were centrifuged on the filters at $12,000 \times \mathrm{g}$ for $20 \mathrm{~min}$ at $4{ }^{\circ} \mathrm{C}$. Remove the supernatant and add TEAB to the solutions and centrifuged at $12,000 \times \mathrm{g}$ for $20 \mathrm{~min}$. The solutions were collected and lyophilized. The lyophilized samples were suspended in TEAB (100 $\mu \mathrm{L}, 50 \mathrm{mM})$ and $40 \mu \mathrm{L}$ of each sample was transferred into new tubes for labeling. Each sample add ITRAQ label reagent (iTRAQ ${ }^{\circledR}$ Reagents-8plex kit, Sigma) following the manufacturer's protocol (Applied Biosystems, Foster 
City, CA, USA). All labeled peptides were pooled together. ITRAQ labeled peptides were fractionated by RP chromatography separation using the 1100 HPLC System(Agilent). RP separation was performed on the Agilent Zorbax Extend RP column ( $5 \mu \mathrm{m}, 150 \mathrm{~mm} \times 2.1 \mathrm{~mm}$ ). Tryptic peptides were separated at an eluent flow rate of $300 \mu \mathrm{L} \cdot \mathrm{min}^{-1}$ and monitored at 210 and $280 \mathrm{~nm}$. Dried samples were harvested from $8 \mathrm{~min}$ to $50 \mathrm{~min}$ and elution buffer were collected in every minute and numbered from 1 to 10 with pipeline. The separated peptides were lyophilized for MS detection[57].

Mass spectrometry analysis. All LC-MS compared with MS analyses were performed on a Q-Exactive mass spectrometer (Thermo, USA) equipped with a Nanospray Flex source (Thermo, USA). The peptides mixtures were loaded by a capillary C18 trap column $(3 \mathrm{~cm} \times 100 \mu \mathrm{m}, \mathrm{C} 18,3 \mu \mathrm{m}, 150 \mathrm{~A})$ and separated by a C18 column $(15 \mathrm{~cm} \times 75 \mu \mathrm{m}, \mathrm{C} 18,3 \mu \mathrm{m}, 120 \mathrm{~A})$ on an ChromXP Eksigent system (AB Sciex). Full MS scans were acquired in the mass range of 300-1600 m compared with a mass resolution of 70,000 and the AGC target value was set at 1,000,000. The 10 most in- tense peaks in MS were fragmented with higher-energy collisional dissociation (HCD) with collision energy of 30 . MS compared with MS spectra were obtained with a resolution of 17,500 with an AGC target of 200,000 and a max injection time of $50 \mathrm{~ms}$. The $\mathrm{Q}-\mathrm{E}$ dynamic exclusion was set for $15.0 \mathrm{~s}$ and run under positive mode[58].

Protein identification and function annotation. Raw data of iTRAQ-labeled proteins by was search against rapa genome protein database in National Center for Biotechnology Information (NCBI) using the Proteome Discoverer TM 2.2 (Thermo, USA). Database searches were performed with trypsin digestion specificity, and the cysteine alkylation was considered as parameters in the database searching. For protein quantification method, iTRAQ 8-plex was chosen. For protein identification, a decoy database search approach was used to determine the false discovery rate (FDR) with acceptance if their FDR $<1.0 \%$ while protein identification containing at least two peptides. The molecular functions of the identified proteins were classified according to their gene ontology annotations and their biological functions. Only the proteins identified with at least two different peptides and $P$ value $<0.05$, and quantified with a ratio of fold change $>1.5$ or fold change $<0.8$ were considered. The NCBI(https://www.ncbi.nlm.nih.gov/) and Uniprot databases (https://www.uniprot.org/) were chosen to the validation and annotation of the protein sequences. Gene Ontology (GO) annotation for the identified proteins was assigned according to Uniprot databas [59].

Statistical analysis. The control and treatment groups were analyzed for statistical significance of differences between multiple groups using one-way ANOVA followed by Duncan's multiple comparisons test. All calculations were performed using SPSS software (version 19.0, IBM, Armonk, NY, USA). All results are presented as mean \pm SD from three independent biological replications. Treatment means were separated by the Duncan multiple range test at $P<0.01$. We use minmax normalization method through the $\mathrm{R}$ programming language to analysis transcriptional and proteomic represent expression values of heat map.

RNA extraction and qPCR analysis of gene expression. There were three biological replicates, and three technical replicates were performed for each gene. The total RNA SAM were completed by using TRNzol Universal Reagent (Tiangen, China), according to the manufacturer's instructions. One microgram of total RNA was used for first-strand cDNA synthesis according to the protocol supplied with PrimeScript $^{\text {TM }}$ RT Master Mix (TaKaRa Biotechnology Dalian, China) and qRT-PCR amplification reactions were performed using a LightCycler®96 Real-Time PCR System (Roche, Basel, Switzerland). Primers used for qRT-PCR assay. The primer sequences for internal standard. The relative gene expression was calculated using the $2-\Delta \Delta \mathrm{Ct}$ method[60]. The primer sequences were designed by Primer-BLAST(https://blast.ncbi.nlm.nih.gov/Blast.cgi ) (Bethesda, MD,USA) based on the sequences of the selected indigenes(Supplementary Table S8). Mean values and standard errors were calculated from three independent experiments with three biological replicates, and relative expression was calculated using the $2-\Delta \Delta \mathrm{Ct}$ method with Actin as the reference.

Author contributions. ZN and WS conceived and designed this study. LL, YP, LM, JW, and YF conducted the experiments. HF, $\mathrm{XL}, \mathrm{CB}$, and $\mathrm{WW}$ analyzed the data. $\mathrm{ZN}$ wrote the manuscript.

Ackowledgments. This study was financially supported by China Agriculture Research System of MOF and MARA (CARS-12); the Agriculture Research System of Gansu Province (GARS-TSZ-1) ; the National Natural Science Foundation of China(NO.31960435) ; the National Natural Science Foundation of China (NO.31860383); the Gansu Provincial Key Laboratory of Aridland Crop Science, Gansu Agricultural University (No.GSCS-2020-Z1).

\section{References}


1. Ding, Y., et al., OST1-mediated BTF3L phosphorylation positively regulates CBFs during plant cold responses. The EMBO Journal, 2018. 37(8): p. e98228.

2. An atypical R2R3 MYB transcription factor increases cold hardiness by CBF-dependent and CBF-independent pathways in apple. New Phytologist, 2018. 218(1).

3. Cheng, G., et al., Transcriptomic Profiling of Young Cotyledons Response to Chilling Stress in Two Contrasting Cotton (Gossypium hirsutum L.) Genotypes at the Seedling Stage. International Journal of Molecular Sciences, 2020. 21(14): $p$. 5095.

4. Bargiela, R., et al., Proteome Cold-Shock Response in the Extremely Acidophilic Archaeon, Cuniculiplasma divulgatum. Microorganisms, 2020. 8(5): p. 759.

5. Dhillon, T., et al., Regulation of Freezing Tolerance and Flowering in Temperate Cereals: The VRN-1 Connection. Plant Physiology, 2010. 153(4): p. 1846-1858.

6. Fowler and B. D. Photoperiod and Temperature Interactions Regulate Low-Temperature-Induced Gene Expression in Barley. Plant Physiology, 2001. 127(4): p. 1676.

7. Vernoux, T., F. Besnard, and J. Traas, Auxin at the shoot apical meristem. Cold Spring Harb Perspect Biol, 2010. 2(4): $p$. a001487.

8. Zhang, J.Z., R.A. Creelman, and J.K. Zhu, From laboratory to field. Using information from Arabidopsis to engineer salt, cold, and drought tolerance in crops. Plant Physiol, 2004. 135(2): p. 615-21.

9. Wang, Y. and H. Li, Identification of proteins associated with paclitaxel resistance of epithelial ovarian cancer using iTRAQbased proteomics. Oncology Letters, 2018. 15: p. 9793.

10. Zhang, N., et al., iTRAQ and virus-induced gene silencing revealed three proteins involved in cold response in bread wheat. Scientific Reports, 2017. 7(1): p. 7524.

11. Vfmab, C., et al., Proteomic variation in Vitis amurensis and V. vinifera buds during cold acclimation. Scientia Horticulturae. 263.

12. Jiang, Q., et al., iTRAQ-based quantitative proteomic analysis of wheat roots in response to salt stress. PROTEOMICS, 2017.

13. Yh, B., et al., iTRAQ protein profile analysis of young and old leaves of cotton (Gossypium hirsutum L.) differing in response to alkali stress - ScienceDirect. Plant Physiology and Biochemistry, 2019. 141: p. 370-379.

14. Thomas, S., et al., ITRAQ-based proteome profiling revealed the role of Phytochrome $A$ in regulating primary metabolism in tomato seedling. Scientific Reports, 2021. 11(1): p. 7540.

15. Mei, et al., ITRAQ-based quantitative analysis reveals proteomic changes in Chinese cabbage (Brassica rapa L.) in response to Plasmodiophora brassicae infection. Scientific reports, 2019. 9(1): p. 12058-12058.

16. Liu, Z., et al., ITRAQ-based quantitative proteomic analysis of salt stress in Spica Prunellae. Scientific Reports, 2019. 9(1): p. 9590.

17. Zl, A., et al., Germinating seed can sense low temperature for the floral transition and vernalization of winter rapeseed (Brassica rapa). Plant Science, 2021. 307.

18. Zeng, X., et al., Identification of cold stress responsive microRNAs in two winter turnip rape (Brassica rapa L.) by high throughput sequencing. Bmc Plant Biology, 2018. 18(1): p. 52.

19. Ma, L., et al., Transcriptome Analysis Reveals Key Cold-Stress-Responsive Genes in Winter Rapeseed (Brassica rapa L.). International Journal of Molecular Sciences, 2019. 20(5).

20. X.Z.Y.X.J.J.F.Z.L.M.D.W.Y.W.W., iTRAQ-Based Comparative Protonic Analysis of the Roots of TWO Winter Turnip Rapes (Brassica rapa L.) with Different Freezing-Tolerance. International journal of molecular sciences, 2018.

21. Muhammad, T., et al., Overexpression of a Mitogen-Activated Protein Kinase SIMAPK3 Positively Regulates Tomato Tolerance to Cadmium and Drought Stress. Molecules, 2019. 24(3).

22. Campos, P.S., et al., Electrolyte leakage and lipid degradation account for cold sensitivity in leaves of Coffea sp plants. Journal of Plant Physiology, 2003. 160(3): p. 283-292.

23. Sairam, R.K., K.V. Rao, and G.C. Srivastava, Differential response of wheat genotypes to long term salinity stress in relation to oxidative stress, antioxidant activity and osmolyte concentration. Plant, 2002. 163(5): p. 1037-1046.

24. Hoekstra, F.A., E.A. Golovina, and J. Buitink, Mechanisms of plant desiccation tolerance. Trends in Plant Science, 2001. 6(9): p. 431-438.

25. Jiang, Y., et al., Micro-encapsulated sodium butyrate attenuates oxidative stress induced by corticosterone exposure and modulates apoptosis in intestinal mucosa of broiler chickens. Animal Production Science, 2014. 55(5): p. 587.

26. Wang, L., et al., Characterization of LhSorP5CS, a gene catalyzing proline synthesis in Oriental hybrid lily Sorbonne: molecular modelling and expression analysis. Botanical Studies, 2017. 58(1): p. 10. 
27. Martinez-Rivas, et al., Differential Contribution of Endoplasmic Reticulum and Chloroplast omega-3 Fatty Acid Desaturase Genes to the Linolenic Acid Content of Olive (Olea europaea) Fruit. Plant and cell physiology, 2016. 57(1).

28. Barrero-Sicilia, C., et al., Lipid remodelling: Unravelling the response to cold stress in Arabidopsis and its extremophile relative Eutrema salsugineum. Plant Science, 2017. 263: p. 194-200.

29. Klíma, M., et al., Dehydrin and proline content in Brassica napus and B. carinata under cold stress at two irradiances. Biologia Plantarum, 2011.

30. Dudler, M.R., Differential induction of distinct g/utathione-S-transferases of wheat by xenobiotics and by pathogen attack. Plant Physiology, 1993. 102(4): p. 1193-1201.

31. Imai, H., et al., Cerebrosides in Seed-Plant Leaves: Composition of Fatty Acids and Sphingoid Bases. 1997: Physiology, Biochemistry and Molecular Biology of Plant Lipids.

32. Yong, W., et al., Integrated proteomic and metabolomic analysis reveals the NADH-mediated TCA cycle and energy metabolism disorders based on a new model of chronic progressive heart failure. Molecular BioSystems, 2013. 9(12): $p$. 3135-3145.

33. Maalekuu, K., et al., The relationship between water loss, lipid content, membrane integrity and LOX activity in ripe pepper fruit after storage. Postharvest Biology \& Technology, 2006. 42(3): p. 248-255.

34. Bak, Cytochromes P450. Arabidopsis Book, 2011. 9: p. e0144.

35. Zhou, G., et al., Silencing OsHI-LOX makes rice more susceptible to chewing herbivores, but enhances resistance to a phloem feeder. Plant Journal, 2010. 60(4): p. 638-648.

36. Paget, C.M., J.M. Schwartz, and D. Delneri, Environmental systems biology of cold-tolerant phenotype in Saccharomyces species adapted to grow at different temperatures. Molecular Ecology, 2015. 23(21): p. 5241-5257.

37. Yang, L.T., et al., Phosphorus-mediated alleviation of aluminum toxicity revealed by the iTRAQ technique in Citrus grandis roots. PLoS ONE, 2019. 14(10): p. e0223516.

38. Min, H., et al., Identification of Drought Tolerant Mechanisms in Maize Seedlings Based on Transcriptome Analysis of Recombination Inbred Lines. Frontiers in Plant Science, 2016. 7: p. 1080-.

39. Taeshin, K., et al., SRXN1 Is Necessary for Resolution of GnRH-Induced Oxidative Stress and Induction of Gonadotropin Gene Expression. Endocrinology, 2019(11): p. 11.

40. Wang, et al., Phylogeny and expression pattern of starch branching enzyme family genes in cassava (Manihot esculenta Crantz) under diverse environments. Molecular and Cellular Biochemistry: An International Journal for Chemical Biology, 2015.

41. Vanlerberghe and Greg, Alternative Oxidase: A Mitochondrial Respiratory Pathway to Maintain Metabolic and Signaling Homeostasis during Abiotic and Biotic Stress in Plants. International Journal of Molecular Sciences, 2013. 14(4): p. 68056847.

42. Vorwerk, S., et al., Enzymatic characterization of the recombinant Arabidopsis thaliana nitrilase subfamily encoded by the NIT2/NIT1/NIT3-gene cluster. Planta, 2001. 212(4): p. 508-516.

43. Xi, C., et al., Mitochondrial proteome during salt stress-induced programmed cell death in rice. Plant Physiology and Biochemistry, 2009. 47(5): p. 407-415.

44. Liu, Y., et al., Physiological and Proteomic Responses of Mulberry Trees (Morus alba. L.) to Combined Salt and Drought Stress. International Journal of Molecular Sciences, 2019. 20(10): p. 2486.

45. Yang, X., et al., Transcriptome profiling of Populus tomentosa under cold stress. Industrial Crops and Products, 2019. 135: p. 283-293.

46. Xu, H.X., X.Y. Li, and J.W. Chen, Comparative transcriptome profiling of freezing stress responses in loquat (Eriobotrya japonica) fruitlets. Journal of Plant Research, 2017.

47. Smith, A.P., et al., Arabidopsis AtGSTF2 is regulated by ethylene and auxin, and encodes a glutathione S-transferase that interacts with flavonoids. Plant Journal, 2010. 36(4): p. 433-442.

48. Guy, C., et al., Metabolomics of temperature stress. Physiologia Plantarum, 2010. 132(2): p. 220-235.

49. Kováčik, J. and M. Bačkor, Changes of phenolic metabolism and oxidative status in nitrogen-deficient Matricaria chamomilla plants. Plant \& Soil, 2007. 297(1-2): p. 255-265.

50. Nawata, S., et al., Evidence of post-translational modification of the tumor suppressor maspin under oxidative stress. International Journal of Molecular Medicine, 2011. 27(2): p. 249.

51. Wang, X., et al., Over-expression of a protein disulfide isomerase gene from Methanothermobacter thermautotrophicus, enhances heat stress tolerance in rice. Gene, 2018. 684

52. Huang, Y., et al., A Brassica napus Reductase Gene Dissected by Associative Transcriptomics Enhances Plant Adaption to Freezing Stress. Frontiers in Plant Science, 2020. 11. 
53. Verma, S. and S.N. Mishra, Putrescine alleviation of growth in salt stressed Brassica juncea by inducing antioxidative defense system. Journal of Plant Physiology, 2005. 162(6): p. 669-677.

54. Gruszka, D., et al., Insights into Metabolic Reactions of Semi-Dwarf, Barley Brassinosteroid Mutants to Drought. International Journal of Molecular Sciences, 2020. 21(5096).

55. Liu, F., et al., Effect of the inoculation of plant growth-promoting rhizobacteria on the photosynthetic characteristics of Sambucus williamsii Hance container seedlings under drought stress. AMB Express, 2019. 9(1): p. 169-.

56. Ramalho, J.C., et al., Stress cross-response of the antioxidative system promoted by superimposed drought and cold conditions in Coffea spp. Plos One, 2018. 13(6): p. e0198694.

57. Wi?Niewski, J.R., et al., Universal sample preparation method for proteome analysis. Nature Methods, 2009. 6(5): p. 359362.

58. Tripathi, A., et al., Erratum for Tripathi et al., "The Cross Talk between TbTim50 and PIP39, Two Aspartate-Based Protein Phosphatases, Maintains Cellular Homeostasis in Trypanosoma brucei". mSphere, 2019. 4(5).

59. Manish L. Raorane, et al. Proteomic insights into the role of the large-effect QTL qDTY 12.1 for rice yield under drought. Molecular Breeding, 2015. 35(6): p. 139.

60. $\mathrm{Hu}, \mathrm{Z}$., et al., Quantitative proteomics identify an association between extracellular matrix degradation and immunopathology of genotype VII Newcastle disease virus in the spleen in chickens. Journal of Proteomics, 2018. 181: $\mathrm{p}$. 201-212. 



\section{Supplementary Files}

This is a list of supplementary files associated with this preprint. Click to download.

- SupplementaryTableS17.xlsx

- SupplementaryTableS8.docx 